\title{
A preliminary study on serological activity of a phenolic glycolipid from Mycobacterium leprae in sera from patients with leprosy, tuberculosis and normal controls
}

\author{
WU QINXUE, YE GANYUN, LI XINYU, LIU QI \\ \& ZHOU LILIN \\ Institute of Dermatology, Chinese Academy of Medical Sciences \\ (Nanjing), China
}

\section{Accepted for publication 16 October 1985}

\begin{abstract}
Summary In this article, we conducted: 1 Study on comparisons of serological activity between phenolic glycolipid (PG1) and its terminal sugar which was a synthetic antigen conjugated to bovine gamma globulin. The sera for comparison were collected from leprosy patients (182 cases), tuberculosis patients (20 cases), and normal persons (108 cases, in non endemic area of leprosy). The results indicated that there was highly significant positive correlation (correlation coefficient, $r=0.72 p<0.0005$ ) between PGl and M-BGG (monosaccharide conjugated to bovine gamma globulin) antigens and their OD values in sera from normal persons similar to those of sera from tuberculosis patients. These suggested that PGI-ELISA and M-BGG-ELISA were highly specific for detection of infection with Mycobacterium leprae. When comparison tests of PGl-ELISA with ML-ELISA were performed, similar sensitivity of the two tests to sera from leprosy patients was found. These tests suggested that PGI-ELISA and M-BGG-ELISA were as highly sensitive as ML-ELISA to sera from leprosy patients. For these reasons, $\mathrm{PGl}-$ and $\mathrm{M}-\mathrm{BGG}-\mathrm{ELISA}$, were both considered highly sensitive and specific for detection of infection with M. leprae. 2 Study on correlation of PGlELISA with ML-ELISA and FLA-ABS.T. The results indicated that they were also highly significant in positive correlation (correlation coefficient, ' $\mathrm{FLA}-$ ABS.T $=0.945 p<0.01 ;{ }^{r}$ ML-ELISA $\left.=0.972 p<0.005\right) .3$ Study on blocking nonspecific binding. Preliminary studies indicated that conventional BSA and GS could be replaced with EA. The efficiency of EA was not only equal to BSA and GS, but also easier to use, much cheaper and easier to get. Therefore, authors suggested popularizing application of EA for blocking nonspecific binding to ML-ELISA and PGI-ELISA or M-BGG-ELISA.
\end{abstract}

\section{Introduction}

The important problem in study of epidemiology in leprosy is the lack of reliable 
technology for detecting subclinical infection with Mycobacterium leprae (ML) and early serodiagnosis of leprosy. It has been well known that FLA-ABS test (FLA-ABS.T) first established by $\mathrm{Abe}^{1}$ was demonstrated as a tool for the abovementioned purposes. ${ }^{2}$ Recently, however, a new simple rapid sensitive and quantitative technology enzyme-linked immunosorbent assay (ELISA) has been developed and widely used in the determination of human humoral antibodies. On the other hand, it has shown that phenolic glycolipid (PGl), a major component of capsule surrounding ML in infected cells, ${ }^{3,4}$ represents the only ML-specific antigen currently available in amounts sufficient for use in large-scale serological screening ${ }^{5}$ and its terminal sugar (M-BGG) has been synthesized. ${ }^{6} \mathrm{At}$ the base of these, the studies on immunodiagnostic of leprosy are increasing rapidly. The present paper describes the relevant results of PG and its terminal sugar, including the blocking agents, with ELISA, and the comparison of PGELISA with FLA-ABS.T in our laboratory.

\section{Materials and Methods}

Antigen preparation. The antigen for FLA-ABS.T is prepared with Wu's technology $;{ }^{7} \mathrm{PGl}$ and $\mathrm{M}-\mathrm{BGG}$ are conveyed to us by Dr Douglas.

Sera (first antibody). Sera from 182 cases of leprosy patients, 20 cases of active tuberculosis patients and 108 cases of healthy persons (nonendemic area of leprosy), and sera for positive and negative control.

Enzyme and fluorescein conjugates (secondary antibody). FITC-IgG was provided by Dr Abe; HPO-IgGMA was provided by Dr Douglas.

Detection of antibodies. It was conducted with Abe's FLA-ABS.T ${ }^{8}$ and Douglas' ELISA ${ }^{9}$ which can be stated briefly as follows: $0.05 \mathrm{ml}$ suspension of antigen was dried onto ' $U$ ' bottom microtitre plates. Antigen-coated wells were blocked to prevent nonspecific binding by adding $0.075 \mathrm{ml}$ of $5 \%$ goat serum (GS) in phosphate buffer saline (PBS), $\mathrm{pH} 7.2$ and incubated overnight at $4^{\circ} \mathrm{C}$. After aspirating the blocking agents, $0.075 \mathrm{ml}$ of serum diluted $1: 200$ in PBS containing $1 \% \mathrm{GS}$ was added to duplicate wells and incubated for $1 \mathrm{hr}$ at $37^{\circ} \mathrm{C}$. After three times washed with PBS, HPO-IgGAM $(0.075 \mathrm{ml}$ of $1: 2000$, diluted with PBS containing $1 \%$ GS) was added and incubated $30 \mathrm{~min}$ at $37^{\circ} \mathrm{C}$. Plates were washed three times with PBS and $0.075 \mathrm{ml}$ of substrate $(0.003 \%$ hydrogen peroxide) and colour reagent $(0.01 \%$ o-phenylenediamine $)$ in citrate buffer $(\mathrm{pH} 5.0)$ were added and incubated in the dark for $30 \mathrm{~min}$ at $37^{\circ} \mathrm{C}$. The reaction was stopped by the addition of $25 \mu \mathrm{l}$ of $4 \mathrm{~N} \mathrm{H}_{2} \mathrm{SO}_{4}$ and read at $490 \mathrm{~nm}$. The test results are reported as the average of duplicate wells. If the difference in OD values of the duplicate wells exceeded $10 \%$ of the mean, the test was repeated.

In the tests for comparison of agents blocking, $5 \%$ GS, $5 \%$ bovine serumalbumin (BSA), 20\% egg-white albumin (EA) and 1\% GS, $1 \%$ BSA, 5\% EA were used in the same plates, and preliminary studies indicated that egg-white 
albumin was at least as efficient as a blocking agent as either goat serum or BSA. Because it is cheaper and more widely available we subsequently used $20 \%$ EA as a blocking agent in the ELISA system. The normal values were calculated with Douglas' formula ${ }^{9}$ as follows:

Negative upper limit $(\mathrm{NUL})=\mathrm{OD}$ value of negative control $+0 \cdot 08 \mathrm{D}$

$\mathrm{D}=\mathrm{OD}$ value of positive control $-\mathrm{OD}$ value of negative control.

On the other hand, we have done Chi-square $\left(\mathrm{X}^{2}\right)$ test, correlation analysis and regression analysis, including significance test of correlation coefficient.

\section{Results}

The results of our tests are shown in Figures 1, 2, 3 and 4 and Table 1. Figures 1 and 3 were used to show individual OD values for ELISA tests against the various classification groups (LL, BL, BB, BT, TT, TB controls, normal controls). Each point represents one individual and the antibody level is expressed as OD value at $490 \mathrm{~nm}$. The short bar in column of each group represents the mean OD value $\left(M_{\mathrm{OD}}\right)$ of each group.

Figure 1(a) shows the results of ML-ELISA. The positivity rates of various groups are: LL $100 \%\left(\mathrm{M}_{\mathrm{OD}} 0 \cdot 47\right), \mathrm{BL} 90 \%\left(\mathrm{M}_{\mathrm{OD}} 0 \cdot 44\right)$, $\mathrm{BB} 100 \%\left(\mathrm{M}_{\mathrm{OD}} 0 \cdot 46\right)$, BT $95.80 \%\left(\mathrm{M}_{\mathrm{OD}} 0 \cdot 48\right)$, TT $75 \%\left(\mathrm{M}_{\mathrm{OD}} 0 \cdot 36\right)$ and Normal controls $1 \%\left(\mathrm{M}_{\mathrm{OD}} 0 \cdot 23\right)$; Figure 1(b) shows the results of PGl-ELISA. The positivity rates of various groups are: LL $86 \cdot 6 \%\left(\mathrm{M}_{\mathrm{OD}} 0 \cdot 47\right)$, BL $93.5 \%\left(\mathrm{M}_{\mathrm{OD}} 0 \cdot 44\right)$, BB $93.3 \%\left(\mathrm{M}_{\mathrm{OD}} 0 \cdot 35\right)$, BT $75 \%\left(M_{\mathrm{OD}} 0 \cdot 34\right)$, TT $33 \cdot 3 \%\left(\mathrm{M}_{\mathrm{OD}} 0 \cdot 20\right)$ and Normal controls $2 \%\left(\mathrm{M}_{\mathrm{OD}} 0 \cdot 17\right)$.
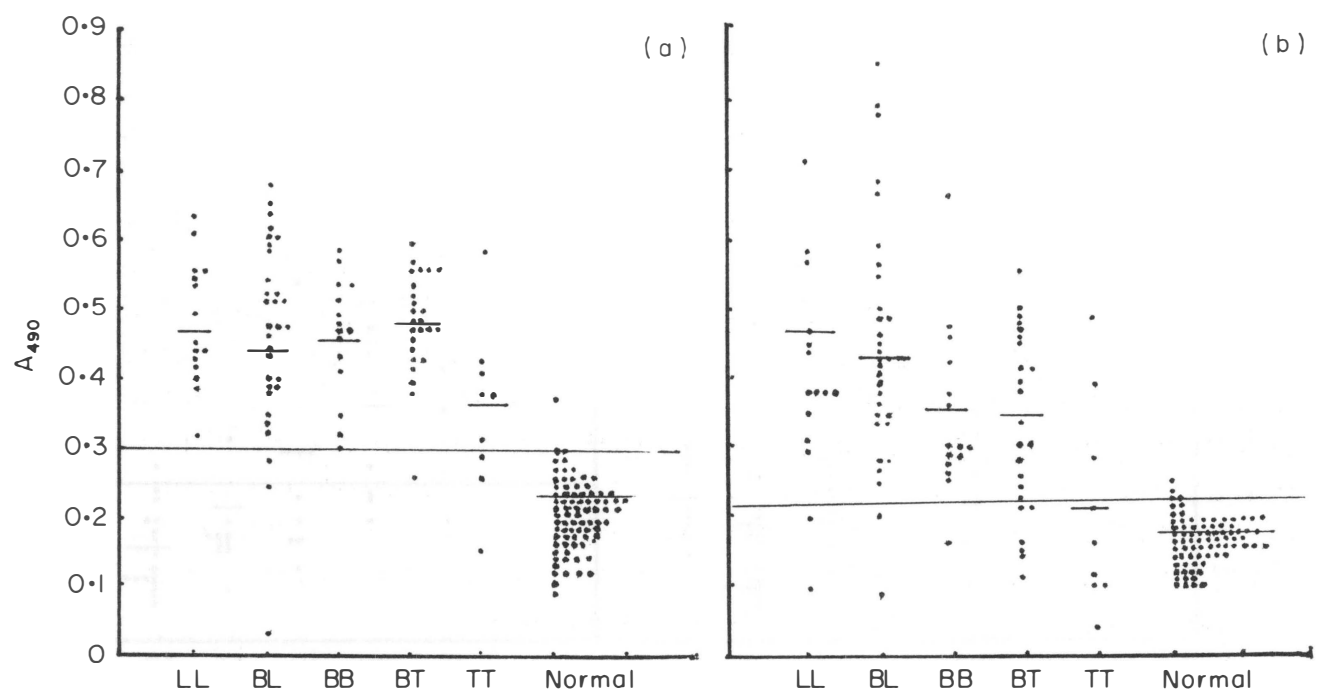

Figure 1. Scattergram of the response of sera from 94 cases of leprosy and 78 normal cases (a) MLELISA, (b) PGI-ELISA. 


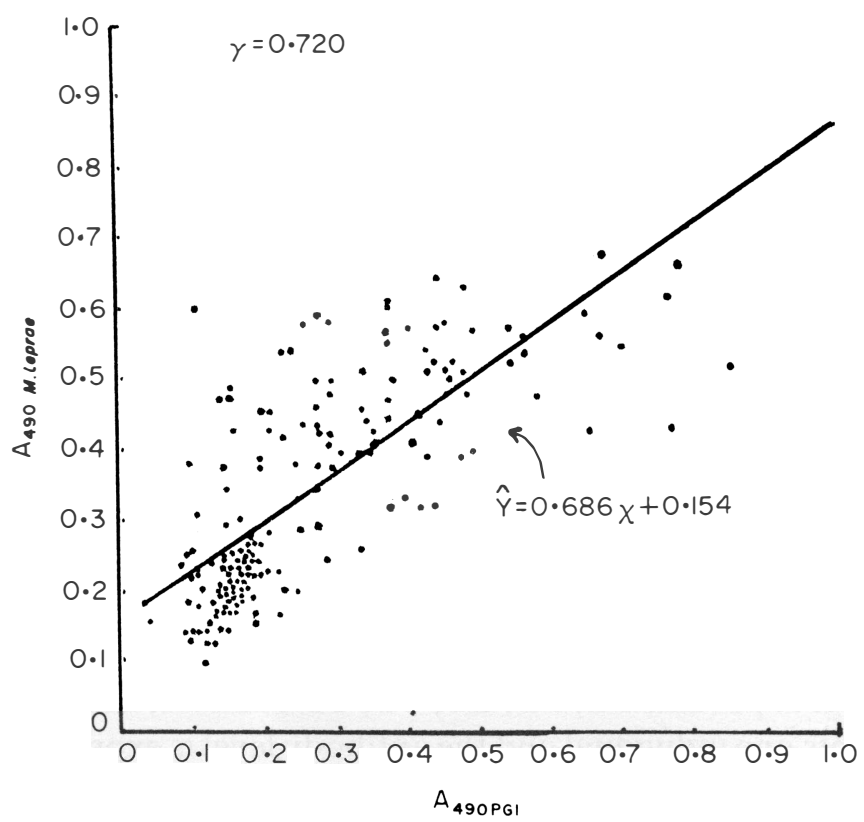

Figure 2. Correlation between ELISA values to the $M$. leprae and PGl in sera from leprosy patients (94) and normal controls (78).
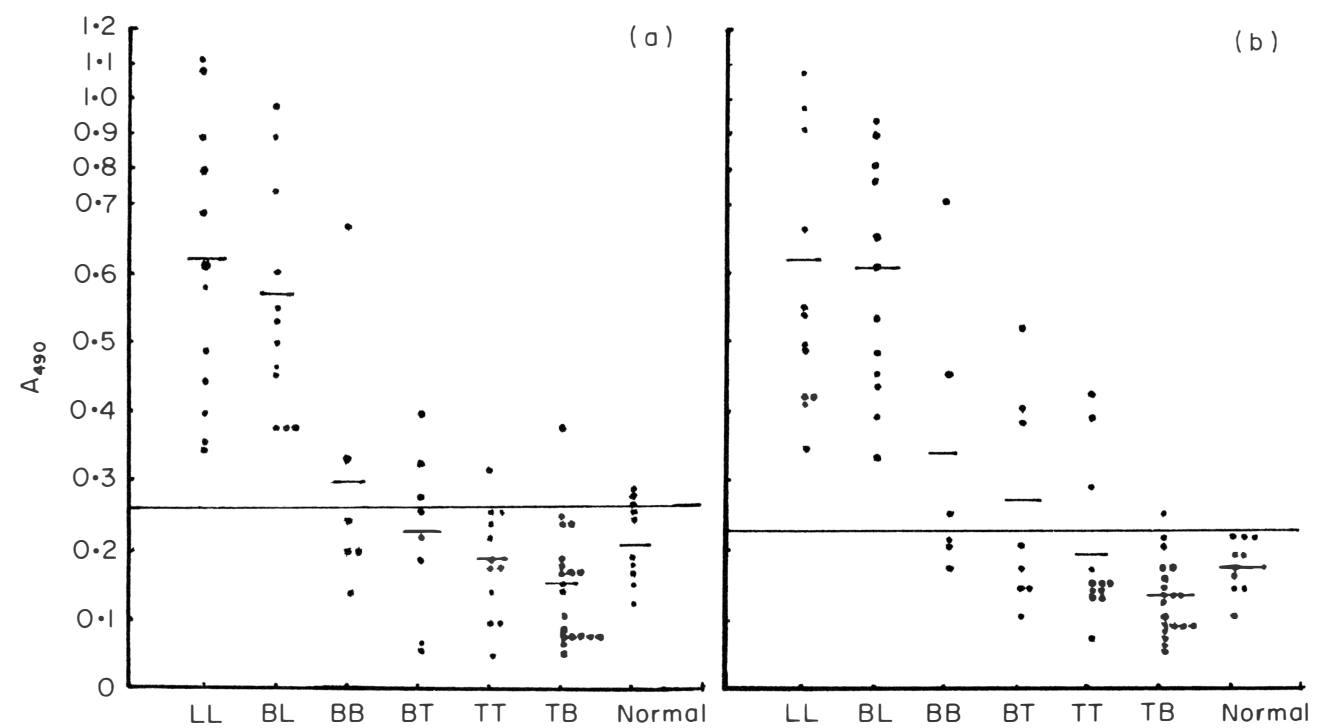

Figure 3. Scattergram of the response of sera from 50 cases of leprosy, 20 cases of tuberculosis and 10 cases of normal control. (a) M-BGG-ELISA, (b) PGl-ELISA. 


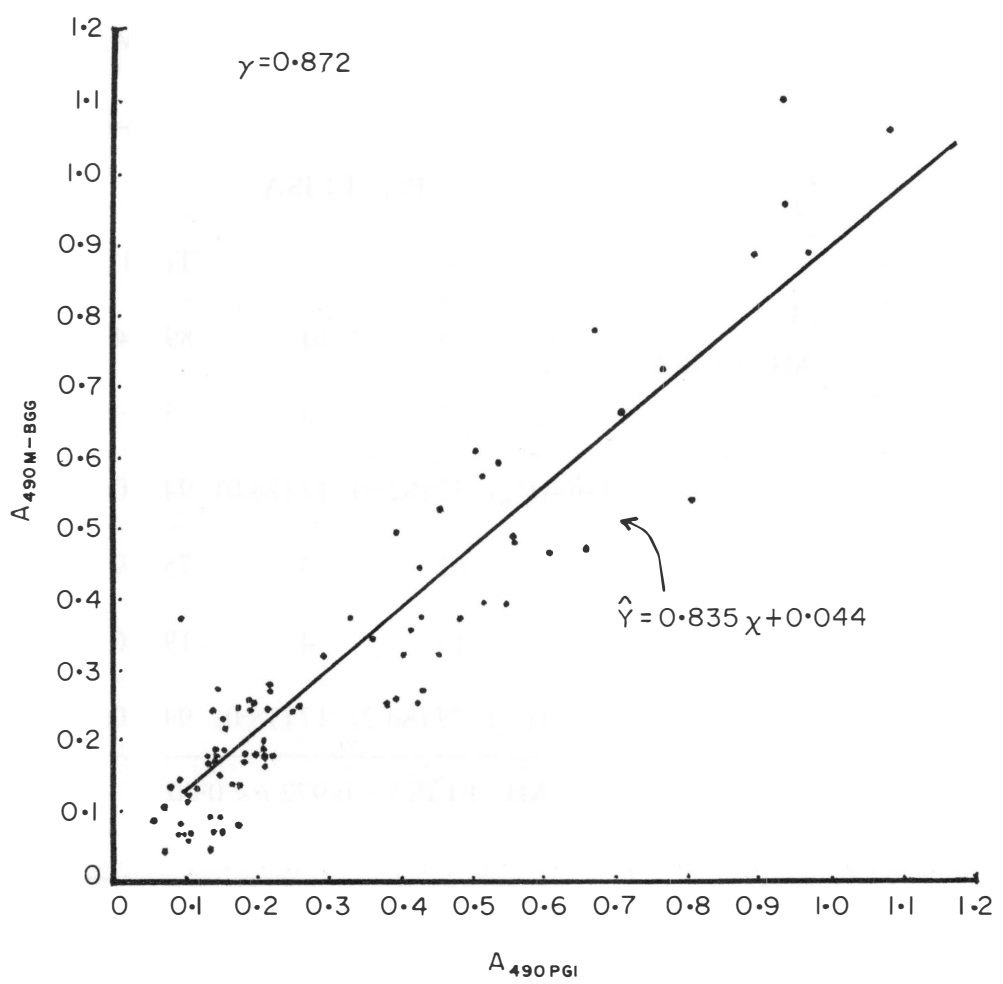

Figure 4. Correlation between values to the $\mathrm{M}-\mathrm{BGG}$ and $\mathrm{PGl}$ in sera from leprosy patients (50), tuberculosis patients (20) and normal controls (10).

From these data: 1 from TT to LL, the content of antibody increased gradually is more regular in PGl-ELISA than in ML-ELISA; 2 from BB to LL, there was essentially the same sensitivity to both antigens, but from TT to BT, the sensitivity is higher in ML-ELISA than in PG-ELISA; and 3 in LL/BL, $\mathrm{M}_{\mathrm{OD}}$ values are essentially equal in both ELISA, but in $\mathrm{BB}, \mathrm{BT} / \mathrm{TT}$, the $\mathrm{M}_{\mathrm{OD}}$ values are higher in ML-ELISA than those in PGl-ELISA. The reason will be further studied.

When ELISA values from a total of 94 leprosy patients and 78 normal controls were analysed (Figure 2), there was a significant correlation between the ML and PG antigens (correlation coefficient, $r=0 \cdot 72, p<0 \cdot 0005$ ). Figure 3 shows the results of (a) M-BGG-ELISA and (b) PGl-ELISA. These data indicate that: 1 from BT to LL, the positivity rates of each group are essentially equal in both of ELISA, and in BL/LL and TB are fairly the same; 2 from TT to LL, the increase of $\mathrm{M}_{\mathrm{OD}}$ values to both antigens are regular; and 3 normal value is higher in $\mathbf{M}_{-}$ BGG-ELISA than in PGl-ELISA, but in TB, the OD values of both ELISA are all lower than those in normal controls.

The correlation analysis (Figure 4) of both ELISA indicated that there was a significant correlation between the PGl and $\mathrm{M}-\mathrm{BGG}$ antigens (correlation coefficient, $r=0 \cdot 872, p<0 \cdot 0005)$. 
Table 1. Results of comparisons of PGl-ELISA with MLELISA and FLA-ABS. (Sera from leprosy patients).

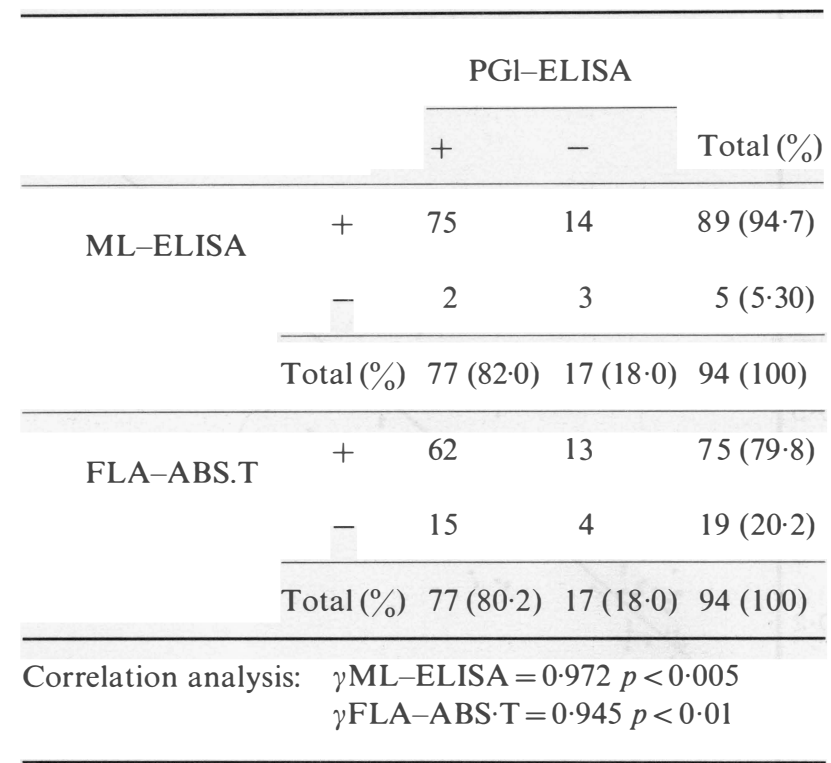

Table 1 shows the results of comparisons of PGl-ELISA with ML-ELISA and FLA-ABS.T, which are obtained from leprosy sera. The positivity percentages are: $94.7 \%$ in ML-ELISA, $82.0 \%$ in PGl-ELISA, $75.8 \%$ in FLAABS.T. Correlation analysis shows that they have highly significant correlation (correlation coefficient, $r_{\text {ML-ELISA }}=0.972 p<0.005 ; r_{\text {FLA-ABS.T }}=0.945 p<0.01$ ).

\section{Discussion}

The comparison of ML-ELISA with PGl-ELISA in sera. From the data in Figures 1 and 3, the reactivity of both antigens to sera at BB, BL/LL patients and Normal controls is the same, but at BT/TT patients, the sensitivity of M. leprae is higher than that of PGl. However, the PGI-ELISA is more specific for infection with $M$. leprae than ML-ELISA, ${ }^{12,13}$ so it may be practical to combine PGlELISA with ML-ELISA for detecting subclinical infection with $M$. leprae, and PGl-ELISA may be more useful for serodiagnosis of multibacillary leprosy.

The comparison of M-BGG and PGl with sera (Figures 2 and 4). Although the negative value was higher in M-BGG-ELISA than in PGI-ELISA, this does not influence the finding in BL/LL patients, and the use of M-BGG has several advantages: approximately six-fold less antigen is required to coat ELISA plates for the same reactivity, ${ }^{11}$ and Tween 20 , a nonanion detergent, may be used in the ELISA. However, PGl and its derivatives are easily removed from ELISA antigen 
plates with Tween 20 . M-BGG is more easily prepared for use in antigen-coating buffers since a sonication step is not required. These results suggest that PGlELISA may be replaced with M-BGG-ELISA, which is more applicable to leprosy endemic areas where the armadillo does not reside. Indeed, the outlook for a synthetic antigen for worldwide application to the serodiagnosis of leprosy looks promising.

Comparison of PGl-ELISA with ML-ELISA and FLA-ABS.T. From the data in Table 4, the ML-ELISA is the most sensitive one, and PGl-ELISA is more sensitive than FLA-ABS.T, the correlation analysis indicated that three methods are highly significant correlation. In fact, we have found FLA-ABS.T with sera from untreated leprosy patients are very sensitive. Moreover, $\mathrm{M}_{\mathrm{OD}}$ values of ELISA are also lower than before. These may be relative to the sera from patients who have received chemotherapy with combinations of three drugs (DDS, RFMP, CLF) for a long period.

In addition, the conventional BSA and GS can be replaced with EA for blocking nonspecific binding. The efficiency is not only equal to BSA and GS, but also facile, much cheaper and easier to get.

In conclusion, although the ML-ELISA and PGl-ELISA methods still cannot find all the paucibacillary patients and the subclinical leprosy infections they are much more sensitive than FLA-ABS.T and excellent for determining multibacillary patients, and PGl-ELISA is more specific for M. leprae than MLELISA and FLA-ABS.T. Additionally, as they are simple, rapid, require little antigen, and provide at least a semiquantitative measurement, it can be said that combinations of ML-ELISA with PGl-ELISA or PGl-ELISA alone may be useful as an epidemiological tool to detect subclinical leprosy infection and in evaluating contacts. Because the test can reflect antibody content, it is possible that it may be useful for following the response of patients to treatment, evaluating the status of disease among patients, and even estimating the degree of infectivity of leprosy patients. As for M-BGG-ELISA, although the normal value presumed in our test is higher, it is still valuable for mentioned purposes, especially in places where there is a shortage of PGl antigen.

\section{Acknowledgments}

We would like to thank Dr Abe for supplying M. vaccae, BCG, cardiolipin and lecithin; Dr Douglas for conveying to us the plates coated with PGl and M-BGG antigens, and Dr Gwinn for his excellent management of the programme.

\section{References}

${ }^{1}$ Abe M, Saito T, Mathur SK. Early Serodiagnosis of Leprosy by Indirect Immunofluorescence. Lepr India, 1976; 48: 272-6. 
2 Report of the Sixth Meeting of the Scientific Working Group on the Immunology of Leprosy, Geneva, 1982: 15.

${ }^{3}$ Brennan PJ, Barrow WW. Evidence for species-specific lipid antigens in Mycobacterium leprae. Int J Lepr, 1980; 48: 382.

${ }^{4}$ Hunter SW, Brennan PJ. A novel phenolic glycolipid from Mycobacterium leprae possibly involved in immunogenicity and pathogenicity. J Bacteriol, 1981; 147: 728-35.

5 Young DB, Buchanan TM. The Phenolic Glycolipid from Mycobacterium leprae: Use in serological tests. Proceedings of the Workshop on Serological Test for Detecting Subclinical Infection in Leprosy, Tokyo; 1983; 19.

${ }^{6}$ Fujiwara T, Hunter SW, Cho SN, Aspinall GO, Brennan PJ. Chemical synthesis and serology of the di- and trisaccharides of the phenolic glycolipid antigens from the leprosy bacillus and preparation of a disaccharide protein conjugate for serodiagnosis of leprosy. Infect Immun, 1984; 43: 245-52.

7 Wu Qinxue et al. Preliminary application of fluorescent leprosy antibody absorption test. Acta Acad Med Sin (in Chinese), 1982; 4, 392-4.

8 Abe $\mathrm{M}$ et al. Fluorescent leprosy antibody absorption (FLA-ABS) test for detecting subclinical infection with Mycobacterium leprae. Int J Lepr, 1980; 48: 109-19.

9 Douglas JT et al. Development of an ELISA for Detection of Antibody in Leprosy. Int J Lepr, 1984; 52: 19-25.

${ }^{10}$ Young DB, Buchanan TM. Detection of Antibodies to the M. leprae Phenolic Glycolipid by Enzyme-Linked Immunosorbent Assay (ELISA). A Personal communication, 1984.

11 Douglas DB et al. Comparison of natural and synthetic antigens for the early detection of leprosy. Joint US-Japan Tuberculosis and Leprosy Symposium, Tokyo: 1984: 143-9.

${ }^{12}$ Cho SN et al. Serological specificity of phenolic glycolipid I from M.leprae and use in serodiagnosis of leprosy. Infect Immun, 1983; 41, 1077-83.

${ }^{13}$ Hunter SW et al. Further specific phenolic glycolipid antigens and a related diacyphthiocerol from M. leprae. J. Biol Chem, 1983; 258: 7556-62. 